A n hang.

\title{
Zur Technik der intravenösen Goldinfusionen und Injektionen.
}

\author{
Von \\ Dr. med. Lautenschläger, \\ I. Assistent der Städtischen Hals- und Nasenklinik in Frankfurt a. M.
}

Mit 3 Abbildungen im Text.

Bei den intravenösen Goldinfusionen und -Injektionen sind genau dieselben Vorsichtsmassregeln $2 u$ beobachten, wie bei jeder intravenösen Einverleibung eines Arzneimittels. Hier seien nur noch einige Einzelheiten erwähnt, die wohl besondere Beachtung verdienen. Die Präparate A und B (Kantharidyläthylendiamin-auri- resp. aurozyanid) wurden zunächst mittelst Zylinders nach Weintraud in starker Verdünnung 1:1000 unter Voraus- und Nachschicken von auf Körpertemperatur erwärmter $0,9 \%$ iger physiologischer Kochsalzlösung infundiert.

Es wurde peinlichste Sorgfalt darauf verwandt, jeden nur möglichen "Wasserfehler" mit Sicherheit auszuschliessen. Als Anfangsdosis gaben wir von Präparat A Dosen von (),02:20,0 bei Frauen und von $0,03: 30,0$ bei Männern - also in 1000 facher Verdünnung und stiegen dann, wenn dies gut ertragen wurde, auf $0,05-0,075$ bis 0,1 , ja sogar bei besonders kräftigen Patienten mit Präparat B, das jetzt ausschliesslich angewandt wird, bis 0,15. Traten jedoch hohe 'Temperaturanstiege oder sonst stärkere Reaktionen' auf, so blieben wir nochmals bei der letzten Dosis, oder gingen wieder auf die vorletzte zurück, setzten unter Umständen auch einen Spritztag ganz aus. Wir haben anfänglich einmal wöchentlich infundiert, sind aber dann, nachdem wir auch ganz kurze 2-3 tägige Intervalle ausprobiert hatten, zu einem viertägigen Turnus übergegangen, weil wir fanden, dass bis dahin meist selbst stärkere Reaktionen abgeklungen waren, und dass dadurch sicher vermierlen wurde, 
dass wir in die reaktive Phase hineinspritzten, ehe der Organismus völlig sein Gleichgewicht wiedererlangt hatte.

Die Infusionen - in Gemeinschaft mit Dr. Senn (II. Assistenten der Klinik) ausgeführt - gingen ohne alle bedenklichen Nebenerscheinungen glatt vonstatten. Bedrohlichere Nebenerscheinungen haben wir danach nie gesehen. Kam einmal etwas von der Lösung ins Unterhautzellgewebe, so bildeten sich wohl Infiltrate, welche schmerzten, aber prompt in wenigen Stundén bis Tagen von selbst oder rascher auf Umschläge von essigsaurer Tonerde zurückgingen. Einige Male beobachteten wir auch vollkommenen Verschluss einer Vene, was uns zunächst den Eindruck einer Thrombose erweckte. Doch gingen auch diese Erscheinungen in kurzer Zeit spontan zurück und die Venen wurden wieder vollkommen durchgängig, so dass von einer ernsteren Gewebsschädigung wohl nicht die Rede sein konnte.

Alle diese Nebenerscheinungen beruhen jedoch nur auf einer mangelnden Infusionstechnik und werden bei einiger Übung fast sicher vermieden werden können. Ich möchte gerade an dieser Stelle erwähnen, dass auch die Notwendigkeit einer Venenfreilegung bei etwa 1500 Infusionen und Injektionen an uns nie herangetreten ist. Bei einiger Sorgfalt ist es sogar fast immer möglich, eine grosse Reihe von Injektionen in ein und dieselbe Vene zu machen, da ein richtig angestochenes Gefäss sich im Laufe mehrerer Infusionen eher noch $\mathrm{zu}$ erweitern und für diese noch günstiger zu werden scheint. Jedenfalls erträgt eine einzige Armvene sämtliche Injektionen (40 und mehr), die zu einer Kur nötig sind, ohne darunter für später auch nur im geringsten zu leiden.

Aus rein praktischen Gründen sind wir in letzter Zeit zur Injektion konzentrierter Lösungen übergegangen. Wir ersparten dadurch dem Patienten die grosse Flüssigkeitsmenge und dem Infundierenden viel Zeit. Nach zahlreichen Versuchen, in denen wir die Konzentrationen immer mehr steigerten, und die uns zeigten, dass man darin sehr weit gehen kann, injizieren wir heute einfach in $2,5 \%$ iger Lösung mit einer Pravazspritze.

Die Injektion gestaltet sich etwa folgendermassen: Patient sitzt auf einem Stuhl, der Arm liegt gerade ausgestreckt auf einem Tischchen, eine Schwester staut und desinfiziert die Ellenbeuge mit Alkohol und Sublimat oder Jodbenzin. Nun wird die Vene mit der Weintraudschen Kanüle punktiert, und nachdem man sich überzeugt, dass die Kanüle einwandsfrei in der Vene sitzt, die Staubinde gelöst. Eine $5 \mathrm{ccm}$ haltende Pravazspritze, in welche zuvor die Goldlösung aufgezogen war, wird alsdann nach gewissenhafter Austreibung aller Luftblasen auf die Kanüle aufgesetzt und langsam entleert. 
Vor dem Herausziehen der Punktionsnadel erfolgt mit dem Finger ein Druck auf die Vene unmittelbar über der Nadelspitze, ein kleiner Handgriff, durch den mit Sicherheit vermieden werden kann, dass beim Herausziehen Goldlösung aus der Vene zurückfliesst, oder dass durch Zurückbluten ein Hämatom entsteht. Danach Heftpflasterverband.

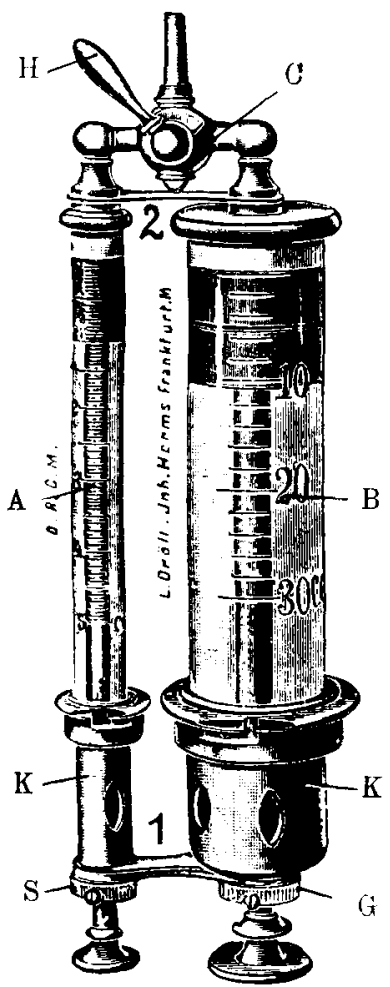

Fig. 1 .
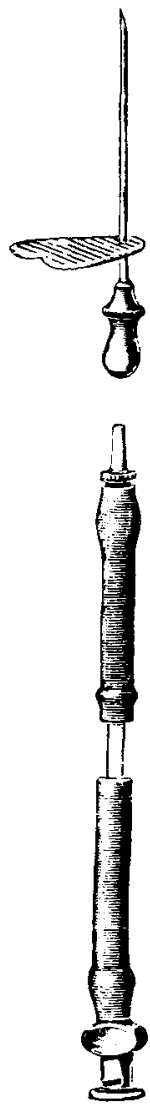

Fig. 2.

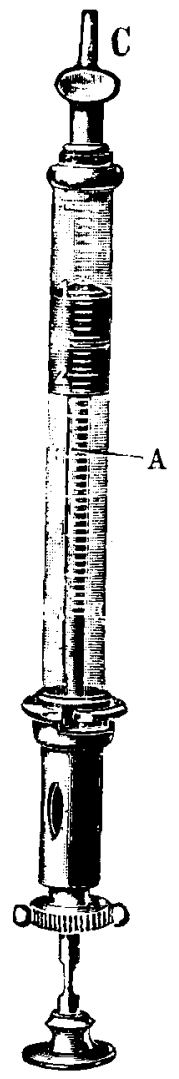

Fig. 3.

I $m 0,025 \mathrm{zu}$ injizieren, bedarf es heute nur noch einer gesamten Irjjektionsmenge von $1 \mathrm{ccm}$, für $0,05-2$, für $0,75-3 \mathrm{ccm}$. Die Lösungen brauchen nicht mehr jedesmal frisch hergestellt zu werden, sie werden jetzt in sterilen Ampullen fertig zum Einspritzen geliefert.

Wie schon gesagt, bedarf es hierfür aber einer absolut sicheren Technik.

Bis diese erlernt ist, dürfte es sich empfehlen, sich einer Spritze $\mathrm{zu}$ bedienen, die es ermöglicht, erst etwas physiologische Kochsalz- 
lösung einzuspritzen, ehe die konzentrierte Goldlösung injiziert wird, und nach dieser abermals etwas Kochsalzlösung nachzuspritzen.

Diese Spritze ist folgendermassen konstruiert:

Wie aus vorhergehender Abbillung ersichtlich, sind zwei Rekordspritzen $\mathrm{A}$ und $\mathrm{B}$ durch die Kuppelung 1 und 2 fest miteinand $\mathrm{r}$ verbunden. Die Kuppelung kann bei 1 durch Umdrehen der beiden Schrauben $S$ und $G$ gelöst werden. Bei 2 sind die beiden Spritzen einfach in das Kombinationsstück $\mathrm{C}$ eingeschraubt, welches dort die Kuppelung darstellt. Dieses Kombinationsstück $\mathrm{C}$ ist so beschaffen, dass es je nach Umlegen des Hahnes $H$ ermöglicht, Flüssigkeit aus derjenigen Spritze austreten zu lassen, welcher der Hahn zugekehrt ist. Der Weg, welchen die Flüssigkeit bei der betreffenden Hahnstellung nimmt, ist aus nebenstehender Zeichnung ersichtlich. Die Grösse der beiden Spritzen ist so gehalten, dass A eine $5 \mathrm{ccm}$ Rekordspritze darstellt, welche es infolge der im Verhältnis zum Querschnitt grossen Länge möglich macht, den Inhalt in kleinsten Mengen ganz langsam austreten zu lassen. Diese Spritze A ist für unsere Zwecke zur Aufnahme der konzentrierten Goldlösung bestimmt. Sie kann natürlich auch einzeln mit einem dazu passenden anschraubbaren Ansatzstück zu jeder beliebigen Injektion bis zu $5 \mathrm{ccm}$ verwendet werden.

Das Volumen der Spritze B beträgt $30 \mathrm{ccm}$. Sie ist in unserem Falle zur Aufnahme der physiologischen Kochsalzlösung bestimmt. Das Volumen ist absichtlich bei dieser Spritze so gross gewählt, damit diese Spritze eventuell auch allein für Kochsalz und andere Infusionen Verwendung finden kann.

Bei $K$ ist an jeder der beiden Spritzen ein Metallgehäuse angebracht, in welches beim Auskochen die Kolben der beiden Stempel zurückgezogen werden können. Das macht jedes Auseinandernehmen der Spritze zum Kochen unnötig.

Der Gang der Injektion für ein beliebiges Mittel, z. B. für unser Gold, würde sich demnach wie folgt gestalten:

Die Spritze ist vollkommen zusammengeschraubt. Der Hahn $\mathrm{H}$ liegt nach $\mathrm{A}$ zu. Es wird durch die gemeinsame Ausflussöffnung Goldlösung aufgezogen, die Luftblasen durch Vorschieben des Spritzenstempels ausgetrieben, dann der Hahn $\mathrm{H}$ nach $\mathrm{B}$ hinübergelegt. Jetzt wird mit $B$ physiologische Kochsalzlösung aufgezogen, nachdem zuvor der Schlauch Sch an das Ausflussende angesetzt worden ist. Die Spritze ist so zur Injektion bereit. In A befindet sich Gold, in B und dem Schlauch $\mathrm{NaCl}$-Lösung. Eine Schwester hält die Spritze senkrecht, das Schlauchende nach unten, damit eventuell Luft, welche sich noch in den Zylindern befindet, nach oben an das entgegen- 
gesetzte Ende der Ausflussöffnung aufsteigt. Nun wird wie bei jeder Venenpunktion die Armvene angestochen, das freie Schlauchende an die Nadel (vermittelst konischen Verschlusses) angesetzt und $\mathrm{NaCl}-$ Lösung injiziert. Hierbei lässt sich sehr leicht konstatieren, ob man sicher in der Vene ist und ob nichts daneben fliesst. Sind so einige Kubikzentimeter richtig injiziert, so lege man den Hahn Hn nach A hinüber und spritze die erforderliche Menge Gold ein. Der Hahn wird dann abermals nach $\mathrm{B}$ zurückgelegt und nochmals $\mathrm{NaCl}$-Lösung nachgespritzt. Dadurch wird das konzentrierte Mittel sogleich verdünnt, und ror allem gelangt beim Herausziehen der Punktionsnadel sicher kein Tropfen der konzentrierten Goldlösung zwischen Venenwand und Haut. Natürlich lasst diese Spritzenkonstruktion die verschiedensten Variationen zu, zumal, wenn man nicht nur zwei, sondern mehrere Spritzen aneinander geschaltet verwendet. 